\title{
Foreword: Mars Science Laboratory, the First Astrobiology Mission to Mars Since Viking
}

\author{
Michael Meyer
}

Published online: 4 September 2012

(C) US Government 2012

The possibility of life on Mars has become a scientific issue of profound importance and of public interest. Since the Viking landings on Mars in 1976, our concepts of the limits to life and our expectation of the habitability of Mars have continued to expand. The modern era of Mars exploration has pursued the strategy of a progressive succession of orbital and landed missions to determine the processes at work on Mars, its record, and its biological potential. Results from these missions have transformed our imagined static lifeless desert planet to a dynamic planet, one that has the potential for life in the past and possibly even the present. Our exploration endeavors culminate with the Mars Science Laboratory (MSL) landing on August 6, 2012. This mission will be the first roving analytical laboratory and considered the first astrobiology mission to Mars since Viking. The rover has ten instruments, including environmental sensors and a laboratory capable of determining elemental composition, mineralogy, and organic content of surface and near-surface samples. This mission will explore Gale Crater, and its $5 \mathrm{~km}$ high central peak, Mount Sharp, whose sediments will provide snapshots of environmental conditions of Mars as it transitioned from its earlier warmer and wetter periods through to a planet whose surface has became cold, dry, and acidic.

The goal of the Mars Science Laboratory and its rover, Curiosity, is to explore a region of Mars and determine if that area was ever able to support microbial life and assess its potential for preservation of biosignatures. The objectives of the mission include: assessing the biological potential of the site by investigating organic compounds, other relevant elements, and biomarkers; characterizing geology and geochemistry, including chemical, mineralogical, and isotopic composition; investigating the role of water, atmospheric evolution, and modern weather/climate; and characterizing the spectrum of surface radiation. This mission is the most complex, sophisticated robotic planetary mission ever attempted and the next step in the Exobiology Strategy for Mars Exploration, as delineated in 1995.

Although conceived before the 2003 launch of the intrepid geological explorers, Spirit and Opportunity, the objectives and capabilities of the planned astrobiology rover have un-

M. Meyer $(\bowtie)$

NASA Headquarters, Washington, D.C., USA

e-mail: Michael.A.Meyer@nasa.gov 
dergone a several transformations. In 2001, a Science Definition Team (SDT), considering the next rover mission to Mars, reported on the potential capabilities of a 2007 Smart Lander. In 2002, the Project Science Integration Group (PSIG), chaired by Dan McCleese and Jack Farmer, was chartered to develop mission scenarios for 2009, with input from the 2001 SDT report and the Mars Exploration Payload Assessment Group. With the challenge of accommodating a lower mission budget, the Group developed a range of potential missions addressing Mars Habitability, Polar Habitability, and Recent Climate. Of these three mission options, Mars Habitability was judged to have the highest science return. However, that mission concept may have been too ambitious and, at least, needed further refinement. The PSIG II was formed with the specific task of developing manageable science requirements for a rover mission addressing habitability. Their report was published in the spring of 2003 and formed the basis of a subsequent Announcement of Opportunity (AO), with instrument proposals due July 2004.

The solicited investigations were to respond to the overall MSL science objective: "to explore and quantitatively assess a local region on the Mars surface as a potential habitat for life, past or present." The request for proposals for in situ instrument investigations was grouped into:

(1) Analytical laboratory investigations that provide and use instruments to analyze Martian atmosphere (gas) samples and/or regolith, rock, ice samples;

(2) Remote sensing investigations that provide and use instruments or suites of instruments to be mounted on the MSL Rover Mast;

(3) Contact instrument investigations that provide and use instruments to be mounted on a robotic arm (or arms) to be provided by MSL; and

(4) Investigations that provide and use individual instruments mounted elsewhere on the MSL Rover including a sensor to assess the radiation environment at the local Martian surface.

In December 2004, eight instruments were selected, which included significant contributions from international partners and an entire instrument from Canadian Space Agency. The selected instruments, combined with two additional instruments contributed by the Russian Space Agency and Spain's Ministry of Science and Technology, formed a powerful integrated and complementary payload for the investigation of past and present environments on Mars.

A critical aspect of the mission portending success is the extensive community involvement in the landing site selection. From over 50 candidate sites, the science community narrowed the number down to a final four, each exhibiting geomorphological and mineralogical evidence of interaction with water. The small landing ellipse and the roving capability of Curiosity have permitted the consideration of extremely promising sites in which the prime science targets are outside the landing ellipse. This includes the selected Gale Crater in which aqueous minerals, presumed characteristic of different epochs of martian history, are the primary targets of the overall exploration strategy. The ability to rove up to and sample across layered deposits, including phyllosilicates and sulfates, will provide critical information for our understanding of the evolution of the martian environment.

Mars Science Laboratory was launched November 26, 2011 and the rover Curiosity landed on Mars August 6, 2012 with a long traverse of potential discovery ahead. 